\title{
Evaluation of Varicella-Zoster Virus Immunoglobulin G in Children With Malignancies on Chemotherapy
}

\author{
Shiva Nazari ${ }^{1, *}$, Mohammad Naderisorki ${ }^{2}$, Shahnaz Armin ${ }^{3}$ and Zari Gholinejad ${ }^{3}$ \\ ${ }^{1}$ Pediatric Congenital Hematologic Disorders Research Center, Research Institute for Children's Health, Shahid Beheshti University of Medical Sciences, Tehran, Iran \\ ${ }^{2}$ Thalassemia Research Center (TRC), Hemoglobinopathy Institute, Mazandaran University of Medical Sciences, Sari, Iran \\ ${ }^{3}$ Pediatric Infections Research Center, Research Institute for Children's Health, Shahid Beheshti University of Medical Sciences, Tehran, Iran \\ "Corresponding author: MD, Pediatric Congenital Hematologic Disorders Research Center, Research Institute for Children's Health, Shahid Beheshti University of Medical \\ Sciences, Tehran, Iran. Email: shnazari2000@gmail.com
}

Received 2020 July 06; Revised 2020 August 16; Accepted 2020 August 26.

\begin{abstract}
Background: Varicella-Zoster Virus (VZV) infection in children with different malignancies on chemotherapy has become an alarming problem. There are insufficient data about VZV seroprevalence among patients in Iran.

Objectives: The current study was conducted to assess the seroprevalence of VZV serum IgG antibodies in children with malignancies on chemotherapy.

Methods: The current single-center cross-sectional study was conducted from January 2018 to December 2019 at Mofid children's hospital, Tehran, Iran. Five milliliters of blood samples were collected from the patients. Serum samples were then tested in duplicate for the IgG antibody against VZV using Varicella-Zoster ELISA IgG/IgM kit.

Results: A total of 54 children with different malignancies were included. Overall, 24 and 30 serum samples were collected from females and males, respectively. The children were five months to 15 -years-old with a mean age of 5.5 years. The overall seroprevalence of VZV IgG in the children was 21.1\% $(n=13 / 54)$. Of patients with positive VZV IgG antibody, $9(69.2 \%)$ were male, and four (30.8\%) were female. Besides, Acute Lymphocytic Leukemia (ALL) with $61.1 \%(n=33 / 54)$ was the most common underlying malignancy among these patients. The prevalence of anti-VZV IgG antibodies was $21.2 \%(n=7 / 33)$ in the group of children with ALL. The highest seroprevalence of the VZV IgG antibody was seen in 11 to 15 -year-old children ( $\mathrm{n}=3 / 7 ; 42.9 \%)$. The prevalence rates of IgG antibodies against VZV among children aged 0-2 years, 3-5 years, and 6-10 years were $28.6 \%, 25.9 \%$, and $7.7 \%$, respectively.

Conclusions: The results showed that children with different malignancies on chemotherapy are quite susceptible to chickenpox infection. It is suggested that a safe and effective live attenuated varicella vaccine in line with the WHO recommendations be incorporated into Iran's national immunization program for children with various malignancies.
\end{abstract}

Keywords: Varicella-Zoster Virus, Seroprevalence, Chemotherapy, Malignancy, ELISA, Iran

\section{Background}

The Varicella-Zoster Virus (VZV) is a member of alphaherpesviruses, extremely infectious, and still endemic in different parts of the world (1). The VZV has no animal reservoir and typically infects humans $(2,3)$. Transmission of VZV primarily occurs in two ways: I) direct contact with skin lesions and II) airborne transmission (4). In most cases, VZV rests chronically in spinal nerves and can target ganglia, epithelial cells, and T lymphocytes (1). This virus can cause two forms of the disease: chickenpox (varicella) and shingles (herpes zoster) $(5,6)$. The infections resulting from VZV exhibit different clinical manifestations such as fever, headache, localized skin lesion, malaise, loss of appetite, rash, retinal necrosis, vasculopathy, and neuropathy (7). Several conditions, such as Human Immunodeficiency Virus (HIV) infection, malignancies (acute lymphoblastic leukemia, neuroblastoma, and adenocarcinoma), immune suppression due to chemotherapy, steroid therapy, old age, and organ transplants are considered as risk factors for VZV infections (6, 8).

Chemotherapy of children with malignancies may decrease the number of T-lymphocytes, B-lymphocytes, and total immunoglobulins (9). Therefore, these patients are highly susceptible to VZV infection. VZV infections in immunosuppressed patients can give rise to various severe abnormalities including hepatic involvement, gastrointestinal diseases, pneumonia, encephalitis, and rarely death (10). It is estimated that at the initial step of chemotherapy, approximately $28 \%$ of pediatric patients 
with acute lymphoblastic leukemia experience VZV (11).

In recent years, the application of live attenuated varicella vaccines and suitable antiviral therapies has reduced related complications and mortality rates from chickenpox. The total varicella-induced mortality rate in most countries is estimated at $0.05 \%$ to $1 \%$ (12). However, the mortality rate from VZV infection is $3 \%$ in pediatric patients with malignancy on chemotherapy without vaccines (11).

\section{Objectives}

In Iran, there is insufficient information about VZV infection in children with different malignancies on chemotherapy. Therefore, this study aimed to assess the VZV immunoglobulin $G$ in this patient group.

\section{Methods}

\subsection{Ethics Statements}

This study was approved by the Ethics Committee of Pediatric Infectious Research Center, Shahid Beheshti University of Medical Sciences, Tehran, Iran.

\subsection{Study Population and Data Collection}

The present single-center cross-sectional study was carried out on children with malignancy on chemotherapy who were hospitalized in Mofid children's hospital from January 2018 to December 2019. A questionnaire was designed for each of the children, and written informed consent was obtained from all patients and their parents during sample collection. Demographic information was gathered, including age, gender, weight, history of vaccination, familial/relative relationship between parents, and clinical manifestations such as fever, rash, history of VZV infection, and the type of underlying malignancy. In general, children with different underlying malignancies such as Acute Lymphocytic Leukemia (ALL), neuroblastoma, Burkitt lymphoma, rhabdomyosarcoma of nasopharynx, hepatoblastoma, adenocarcinoma, juvenile myelomonocytic leukemia, nephroblastoma, and lymphadenopathy were included in the present study.

\subsection{Determination of VZV-specific IgG antibodies by ELISA}

In the present study, five milliliters of blood samples were taken from children with malignancies on chemotherapy. The blood samples were centrifuged at $1,300 \mathrm{~g}$ for $10 \mathrm{~min}$, and serum samples were separated. Then, they were kept at $-20^{\circ} \mathrm{C}$ before further analysis. They were tested in duplicate for the IgG antibody against VZV using the Varicella-Zoster ELISA IgG/IgM kit (Vircell, S.L. Santa Fe, Granada, Spain). Briefly, for the IgG test, $100 \mu \mathrm{l}$ of serum diluent was added to all wells. At the next step, 5 $\mu \mathrm{l}$ of each sample, $5 \mu \mathrm{l}$ of positive control, $5 \mu \mathrm{l}$ of negative control, and $5 \mu \mathrm{l}$ of cutoff control (in duplicate) were added to the corresponding wells. Each of the wells was covered with a sealing sheet and incubated at $37^{\circ} \mathrm{C}$ for $45 \mathrm{~min}$. Following incubation, all wells were washed five times with $0.3 \mathrm{ml}$ of washing solution per well. At the next step, $100 \mu \mathrm{l}$ of IgG conjugate solution was immediately added to each well, the wells were covered with a sealing sheet and incubated at $37^{\circ} \mathrm{C}$ for $30 \mathrm{~min}$. They were then washed separately five times with $0.3 \mathrm{ml}$ of washing solution, and $100 \mu \mathrm{l}$ of substrate solution was immediately added to each well. The wells were incubated at room temperature for $20 \mathrm{~min}$ and shielded from light. At the final step, $50 \mu \mathrm{l}$ of stopping solution was added immediately to each well, and the wells were read with a spectrophotometer at $450 / 620 \mathrm{~nm}$ within one hour of stopping. Vircell IgG positive and negative controls containing Neolone and Bronidox, , were commercially included in the Varicella-Zoster ELISA IgG/IgM kit. All of the previous steps and the process of determining the cutoff amount were carried out as per the instructions of the manufacturer.

\subsection{Statistical Analysis}

Information of all patients was collected from the hospital database. A descriptive analysis was conducted using SPSS 23 Package program (SPSS Inc., Chicago, IL, USA).

\section{Results}

\subsection{Demographic Data and Characteristics of Children With Malignancies}

In this research, a total of 54 children with different malignancies were studied from January 2018 to December 2019 at the Mofid children's hospital, Tehran, Iran. Data on demographics and VZV seroprevalence among these patients are summarized in Table 1. Overall, 24 serum samples were collected from females and 30 serum samples from males. Among different malignancies, ALL with $61.1 \%$ (n $=33 / 54)$ and neuroblastoma with $9.3 \%(n=5 / 54)$ were the most common underlying malignancies among the patients. The results showed that nine (16.7\%) children were born from parents with consanguineous marriage.

In general, the mean weight of the children was $18 \pm$ $0.2 \mathrm{~kg}$, in the range of 5 to $58 \mathrm{~kg}$. The age of the children ranged from 5 months to 15 years, with a mean age of $5.5 \pm$ 0.5 years. Among the studied participants, 50\% $(n=27 / 54)$ and $24.1 \%(n=13 / 54)$ were in the age range of 3 to 5 years and 6 to 10 years, respectively. The mean age of females was $4.6 \pm 8.7$, within the age group of 5 months to 15 years. On the other hand, the age of males ranged from 1 to 14 years, 


\begin{tabular}{|c|c|c|c|}
\hline Demographic information & $\begin{array}{l}\text { Number of all participants: } 54 \\
\qquad(\mathbf{1 0 0} \%)\end{array}$ & $\begin{array}{l}\text { Number of anti-VZV IgG-positive } \\
\text { subjects: } 13(24.1 \%)\end{array}$ & $\begin{array}{c}\text { Number of anti-VZV IgG-negative } \\
\text { subjects: } 41(75.9 \%)\end{array}$ \\
\hline \multicolumn{4}{|l|}{ Gender } \\
\hline Female & $24(44.4 \%)$ & $4(16.7 \%)$ & $20(83.3 \%)$ \\
\hline Male & $30(55.6 \%)$ & $9(30 \%)$ & $21(70 \%)$ \\
\hline \multicolumn{4}{|l|}{ Age groups (years) } \\
\hline $0-2$ & $7(13 \%)$ & $2(28.6 \%)$ & $5(71.4 \%)$ \\
\hline 5-Mar & $27(50 \%)$ & $7(25.9 \%)$ & $20(74.1 \%)$ \\
\hline 10-Jun & $13(24.1 \%)$ & $1(7.7 \%)$ & $12(92.3 \%)$ \\
\hline $15-\mathrm{Nov}$ & $7(13 \%)$ & $3(42.9 \%)$ & $4(57.1 \%)$ \\
\hline \multicolumn{4}{|l|}{ Weight (kg) } \\
\hline $1-10$ & $5(9.3 \%)$ & $1(20 \%)$ & $4(80 \%)$ \\
\hline $11-15$ & $18(33.3 \%)$ & $5(27.8 \%)$ & $13(72.2 \%)$ \\
\hline $16-20$ & $13(24.1 \%)$ & $4(30.8 \%)$ & $9(69.2 \%)$ \\
\hline $21-30$ & $11(20.4 \%)$ & $1(9.1 \%)$ & $10(90.9 \%)$ \\
\hline$>30$ & $7(13 \%)$ & $2(28.6 \%)$ & $5(71.4 \%)$ \\
\hline \multicolumn{4}{|l|}{ Vaccination } \\
\hline complete & $33(61.1 \%)$ & $6(18.2 \%)$ & $27(81.8 \%)$ \\
\hline incomplete & $21(38.9 \%)$ & $7(33.3 \%)$ & $14(66.7 \%)$ \\
\hline \multicolumn{4}{|l|}{ Malignancy } \\
\hline ALL & $33(61.1 \%)$ & $7(21.2 \%)$ & $26(78.8 \%)$ \\
\hline Neuroblastoma & $5(9.3 \%)$ & $2(40 \%)$ & $3(60 \%)$ \\
\hline Burkitt lymphoma & $1(1.9 \%)$ & $0(0 \%)$ & $1(100 \%)$ \\
\hline $\begin{array}{l}\text { Rhabdomyosarcoma of } \\
\text { nasopharynx }\end{array}$ & $1(1.9 \%)$ & $0(0 \%)$ & $1(100 \%)$ \\
\hline Hepatoblastoma & $1(1.9 \%)$ & $0(0 \%)$ & $1(100 \%)$ \\
\hline Port Catheter & $1(1.9 \%)$ & $0(0 \%)$ & $1(100 \%)$ \\
\hline Hypospadias & $1(1.9 \%)$ & $0(0 \%)$ & $1(100 \%)$ \\
\hline Adenocarcinoma & $2(3.7 \%)$ & $1(50 \%)$ & $1(50 \%)$ \\
\hline JML & $1(1.9 \%)$ & $0(0 \%)$ & $1(100 \%)$ \\
\hline Nephroblastoma & $2(3.7 \%)$ & $0(0 \%)$ & $2(100 \%)$ \\
\hline Gastroenteritis & $1(1.9 \%)$ & $1(100 \%)$ & $0(0 \%)$ \\
\hline Lung cancer & $1(1.9 \%)$ & $0(0 \%)$ & $1(100 \%)$ \\
\hline Lymphadenopathy & $1(1.9 \%)$ & $1(100 \%)$ & $0(0 \%)$ \\
\hline CML & $1(1.9 \%)$ & $1(100 \%)$ & $0(0 \%)$ \\
\hline AML & $1(1.9 \%)$ & $0(0 \%)$ & $1(100 \%)$ \\
\hline Frenulum of tongue & $1(1.9 \%)$ & $0(0 \%)$ & $1(100 \%)$ \\
\hline \multicolumn{4}{|l|}{ Fever } \\
\hline Yes & $21(38.9 \%)$ & $2(9.5 \%)$ & $19(90.5 \%)$ \\
\hline No & $33(61.1 \%)$ & $11(33.3 \%)$ & $22(66.7 \%)$ \\
\hline \multicolumn{4}{|l|}{ Rash and erythema } \\
\hline Yes & $8(14.8 \%)$ & $2(25 \%)$ & $6(75 \%)$ \\
\hline No & $46(85.2 \%)$ & $11(23.9 \%)$ & $35(76.1 \%)$ \\
\hline \multicolumn{4}{|l|}{ History of VZV infection } \\
\hline Yes & $2(3.7 \%)$ & $2(100 \%)$ & $0(0 \%)$ \\
\hline No & $52(96.3 \%)$ & $11(21.2 \%)$ & $41(78.8 \%)$ \\
\hline
\end{tabular}

Abbreviations: JML: Juvenile Myelomonocytic Leukemia, AML: Acute Myeloid Leukemia, CML: Chronic Myelogenous Leukemia, ALL: Acute Lymphocytic Leukemia. 
with a mean age of $6.1 \pm 3.3$ years. Among the 54 children, $33(61.1 \%)$ had a previous history of vaccination against varicella. Moreover, two (3.7\%) patients had a previous history of VZV infection. In general, analyses indicated that 38.9\% $(n=21 / 54)$ of the children had a fever and $14.8 \%(n=8 / 54)$ had rash and erythema.

\subsection{Serum Findings}

Generally, the results of the ELISA test indicated that the overall seroprevalence of VZV IgG was 21.1\% $(n=13 / 54)$ in children with malignancies on chemotherapy. The seroprevalence of VZV among sex groups and age groups (below 15 years) is shown in Table 1 and Figure 1 . Of the patients with positive VZV IgG antibody, nine (69.2\%) were males, and four (30.8\%) were females. The rate of VZV infection was higher in males than in females. Male and female percentages for VZV infection were 30\% $(n=9 / 30)$ and $16.7 \%(n=4 / 24)$, respectively. The highest seroprevalence of VZV IgG antibody was seen in children aged 11 to 15 years $(n=3 / 7 ; 42.9 \%)$. On the other hand, the lowest seroprevalence of VZV IgG antibody was seen in children aged 6 to 10 years $(n=1 / 13 ; 7.7 \%)$. The prevalence rates of IgG antibodies against VZV among age groups of 0-2 years, 3-5 years, 6-10 years, and $11-15$ years were $28.6 \%, 25.9 \%, 7.7 \%$, and $42.9 \%$, respectively. The prevalence of VZV IgG antibodies was low (9.1\%, $n=1 / 11)$ among children with weights ranging between 21 and $30 \mathrm{~kg}$. Moreover, the seropositivity of anti-VZV IgG was higher in unvaccinated children (33.3\%, $\mathrm{n}=7 / 21)$ than in vaccinated children $(18.2 \%, \mathrm{n}=6 / 32)$ (children over four-years-old who had received perfect national immunization). The prevalence of anti-VZV IgG antibodies was $21.2 \%(n=7 / 33)$ in the group of children with ALL. This group included four (57.1\%) males and three (42.9\%) females within the age group of 1.5 to 15 years. Furthermore, the prevalence of anti-VZV IgG antibodies was $40 \%(n=2 / 5)$ in the group of children with neuroblastoma. This group included one (50\%) male and one (50\%) female within the age group of 1.5 to 5 years. The prevalence of anti-VZV IgG antibodies was $100 \%(n=2 / 2)$ among patients with a history of VZV infection.

\section{Discussion}

In general, VZV is transmitted through close personal contact or airborne droplets (11). Children are susceptible to VZV infection, and it is estimated that approximately $90 \%$ of VZV infections are diagnosed in children aged $<$ 15 years worldwide $(13,14)$. Children on chemotherapy are more susceptible to VZV infection (15). This study was carried out to survey the seroprevalence of anti-VZV IgG antibodies in children with underlying malignancies on chemotherapy. In this study, 13 out of 54 children (21.1\%) (age 0-15 years) were seropositive. There are various reports of the prevalence of VZV antibodies among different groups of patients and healthy subjects in different countries. In 2020, Zawitkowska et al. found that $29.4 \%$ of the pediatric population with ALL were seropositive (9). Luan et al. revealed that among 3,014 studied children in China, more than $54 \%$ with varicella vaccination were anti-VZV IgG-positive (16). In 2016, Ojha et al. found that the incidence rate of VZV infection was $2.3 \%$ among patients with pediatric cancer in developing countries (17). In a nationwide population-based cohort study, Lin et al. reported that children with cancer had a higher risk of contracting VZV infection, the incidence rate of which was higher in children with cancer than in those in the non-cancer cohort (20.7 vs. 2.4 per 10,000 person-years; IRR $=8.6$; $95 \%$ $\mathrm{CI}=4.8-15.6)$ (18). In Iran, Fahimzad et al. revealed that among 570 healthy individuals, 393 subjects were tested positive for VZV IgG (19). In the present study, most of the children were diagnosed with ALL, and the results revealed that they faced a high risk of VZV infection. Therefore, immunization against VZV is required to prevent virus infection and transmission. Given that the use of various drugs, such as steroids, leads to the suppression of the immune system in patients, VZV infection among children with different malignancies such as ALL, neuroblastoma, Acute Myeloid Leukemia (AML), and Chronic Myelogenous Leukemia (CML) may occur more frequently than in healthy children.

Moreover, it is predicted that VZV infection can cause many problems during the treatment and recovery of the patients $(8,10,18)$. In the current national immunization program in Iran (Sep. 2012), vaccination against VZV is conducted only among high-risk groups (20). However, it is recommended that vaccination against VZV in these patients be conducted following the completion of chemotherapy and the full recovery of the immune system (17). The highest seroprevalence of VZV IgG antibody was seen in 11- to 15-year-old children. The obtained results are in agreement with those of previous studies conducted in China and the USA, which reported that the prevalence of VZV IgG antibodies was high among children aged 7 to 12 years and $>10$ years, respectively $(17,18)$. However, this result is not consistent with those of published studies by Fahimzad et al. in Iran (19) and Luan et al. in China (16). These two studies found that the VZV seropositivity was $>90 \%$ and $50 \%$ in individuals aged $>19$ years and 4 to 6 years, respectively. The divergence observed in the prevalence and incidence outcomes of studies conducted in different countries may be due to factors such as 1 ) different sample sizes and methods used to detect VZV, 2) varying public knowledge/awareness levels, 3) different hygienic 


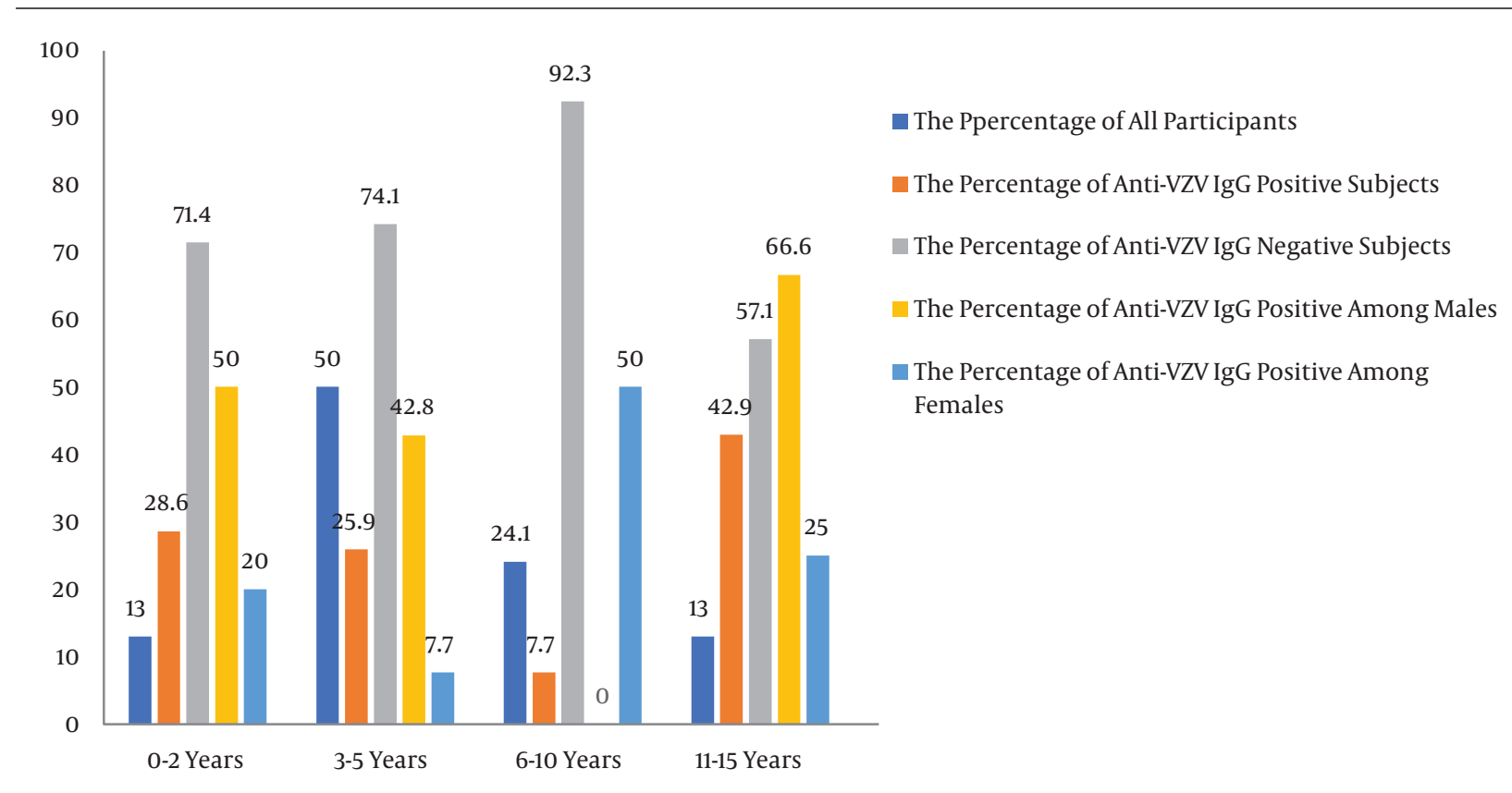

Figure 1. Prevalence of anti-VZV IgG antibody according to gender and age in children with malignancy on chemotherapy in Tehran, Iran. The figure legends show the percentage of VZV seropositivity among different age groups.

conditions of countries, 4) social and demographic differences, and 5) different immunization programs (17).

However, our study has several limitations. First, the number of included patients and the sample size were small. Of note, a single children's hospital was considered only for research; thus, the results might not be generalizable to all hospitals in Tehran. Second, constrained by low budget, we could not apply molecular methods to detect VZV in samples, and thus, this research remained limited in scope to analyzing the seroprevalence of anti-VZV IgG in children. Finally, we could not analyze the association between treatment strategies for childhood malignancies and VZV infection.

In conclusions, according to the results of this study, the seroprevalence of VZV was 21.1\% in children with malignancy on chemotherapy. This incidence rate showed that these patients are highly susceptible to chickenpox infection. Moreover, these results illustrated that the applied therapeutic approach was not suitable and effective for the patients. Therefore, it is suggested that, based on recommendations of the World Health Organization (WHO), a safe and effective live attenuated varicella vaccine be incorporated into the national immunization program for children with different malignancies in Iran.

\section{Acknowledgments}

We would like to thank the Pediatric Infections Research Center, Shahid Beheshti University of Medical Sciences, Tehran, Iran for kind cooperation.

\section{Footnotes}

Authors' Contribution: Shiva Nazari and Mohammad Naderisorki: Conceptualization, data curation, formal analysis, and writing the original draft. Shiva Nazari and Shahnaz Armin: Conceptualization, methodology, project administration, and writing the original draft. Shiva Nazari and Zari Gholinejad: Data curation, formal analysis, writing the original draft, writing, review, and editing. Shahnaz Armin and Mohammad Naderisorki: Language editing.

Conflict of Interests: All of the authors declare that there are no commercial, personal, political, or any other potential conflicting interests related to the submitted manuscript.

Ethical Approval: This study was approved by the Ethics Committee of Pediatric Infectious Research Center, Shahid Beheshti University of Medical Sciences, Tehran, Iran.

Funding/Support: This research did not receive any specific grant from funding agencies in public, commercial, or not-for-profit sectors. 
Informed Consent: All authors made substantial contributions to study conception and design and data acquisition, analysis, or interpretation. They played an active role in drafting the article or revising it critically for important intellectual content, gave the final approval of the version to be published, and agreed to be accountable for all aspects of the work. Written informed consent was obtained from all patients and their parents during sample collection.

\section{References}

1. Gershon AA, Breuer J, Cohen JI, Cohrs RJ, Gershon MD, Gilden D, et al. Varicella zoster virus infection. Nat Rev Dis Primers. 2015;1:15016. doi: 10.1038/nrdp.2015.16. [PubMed: 27188665]. [PubMed Central: PMC5381807].

2. Gilden D, Cohrs RJ, Mahalingam R, Nagel MA. Neurological disease produced by varicella zoster virus reactivation without rash. Curr Top Microbiol Immunol. 2010;342:243-53. doi: 10.1007/82_2009_3. [PubMed: 20186614]. [PubMed Central: PMC3076592].

3. Gershon AA, Gershon MD. Pathogenesis and current approaches to control of varicella-zoster virus infections. Clin Microbiol Rev. 2013;26(4):728-43. doi: 10.1128/CMR.00052-13. [PubMed: 24092852]. [PubMed Central: PMC3811230].

4. Lachiewicz AM, Srinivas ML. Varicella-zoster virus post-exposure management and prophylaxis: A review. Prev Med Rep. 2019;16:101016. doi: 10.1016/j.pmedr.2019.101016. [PubMed: 31890472]. [PubMed Central: PMC6931226]

5. Speth F, Hinze $\mathrm{CH}$, Andel S, Mertens T, Haas JP. Varicella-zostervirus vaccination in immunosuppressed children with rheumatic diseases using a pre-vaccination check list. Pediatr Rheumatol Online J. 2018;16(1):15. doi: 10.1186/s12969-018-0231-3. [PubMed: 29499726]. [PubMed Central: PMC5833060].

6. Lai YL, Su YC, Kao CH, Liang JA. Increased risk of varicella-zoster virus infection in patients with breast cancer after adjuvant radiotherapy: A population-based cohort study. PLoS One. 2019;14(1). e0209365. doi: 10.1371/journal.pone.0209365. [PubMed: 30625182]. [PubMed Central: PMC6326420].

7. Mueller NH, Gilden DH, Cohrs RJ, Mahalingam R, Nagel MA. Varicella zoster virus infection: clinical features, molecular pathogenesis of disease, and latency. Neurol Clin. 2008;26(3):675-97. viii. doi: 10.1016/j.ncl.2008.03.011. [PubMed: 18657721]. [PubMed Central: PMC2754837].

8. Duzgol M, Ozek G, Bayram N, Oymak Y, Kara A, Demirag B, et al. Varicella-Zoster Virus Infections in Pediatric Malignancy Patients: A Seven-Year Analysis. Turk J Haematol. 2016;33(4):346-8. doi: 10.4274/tjh.2016.0046. [PubMed: 27751970]. [PubMed Central: PMC5204192 financial interests, relationships, and/or affiliations relevant to the subject matter or materials included].
9. Zawitkowska J, Lejman M, Szmydki-Baran A, Zaucha-Prażmo A, Czyżewski K, Dziedzic M, et al. Varicella-zoster virus infection in the pediatric population with acute lymphoblastic leukemia in Poland Journal of Medical Virology. 2020.

10. Noronha V, Ostwal V, Ramaswamy A, Joshi A, Nair R, Banavali SD, et al. Chicken pox infection in patients undergoing chemotherapy: A retrospective analysis from a tertiary care center in India. J Infect Public Health. 2017;10(1):8-13. doi: 10.1016/j.jiph.2015.12.016. [PubMed: 26970699].

11. Kim SK, Kim MC, Han SB, Kim SK, Lee JW, Chung NG, et al. Clinical characteristics and outcomes of varicella zoster virus infection in children with hematologic malignancies in the acyclovir era. Blood Res. 2016;51(4):249-55. doi: 10.5045/br.2016.51.4.249. [PubMed: 28090487]. [PubMed Central: PMC5234238].

12. van de Wetering MD, Vossen MT, Jansen MH, Caron HN, Kuijpers TW. Varicella vaccination in pediatric oncology patients without interruption of chemotherapy. J Clin Virol. 2016;75:47-52. doi: 10.1016/j.jcv.2016.01.004. [PubMed: 26780112].

13. Ma H, Fontaine R, Centers for Disease Control, Prevention. Varicella outbreak among primary school students-Beijing, China, 2004. MMWR Morb Mortal Wkly Rep. 2006;55(Suppl 1):39-43.

14. Yang Y, Geng X, Liu X, Wang W, Zhang J. Association between the incidence of varicella and meteorological conditions in Jinan, Eastern China, 2012-2014. BMC Infect Dis. 2016;16:179. doi: 10.1186/s12879-0161507-1. [PubMed: 27102884]. [PubMed Central: PMC4840874].

15. Wiegering V, Schick J, Beer M, Weissbrich B, Gattenlohner S, Girschick $\mathrm{HJ}$, et al. Varicella-zoster virus infections in immunocompromised patients - a single centre 6-years analysis. BMC Pediatr. 2011;11:31. doi: 10.1186/1471-2431-11-31. [PubMed: 21569228]. [PubMed Central: PMC3115870].

16. Luan L, Shen X, Qiu J, Jing Y, Zhang J, Wang J, et al. Seroprevalence and molecular characteristics of varicella-zoster virus infection in Chinese children. BMC Infect Dis. 2019;19(1):643. doi: 10.1186/s12879-0194233-7. [PubMed: 31324226]. [PubMed Central: PMC6642589].

17. Ojha RP, Stallings-Smith S, Aviles-Robles MJ, Gomez S, Somarriba MM Caniza MA. Incidence and case-fatality of varicella-zoster virus infection among pediatric cancer patients in developing countries. Eur J Pediatr. 2016;175(4):581-5. doi: 10.1007/s00431-015-2672-8. [PubMed: 26608932].

18. Lin $\mathrm{HC}$, Chao $\mathrm{YH}, \mathrm{Wu} \mathrm{KH}$, Yen TY, Hsu YL, Hsieh TH, et al. Increased risk of herpes zoster in children with cancer: A nationwide populationbased cohort study. Medicine (Baltimore). 2016;95(30). e4037. doi 10.1097/MD.0000000000004037. [PubMed: 27472677]. [PubMed Central: PMC5265814].

19. Fahimzad A, Nouri M, Shiva F, Shamshiri AR, Gholinejad Z, Karimi A. The Seroprevalence of Varicella Zoster Virus in a Healthy Population from Tehran, Iran. Archives of Pediatric Infectious Diseases. 2018; In Press(In Press). doi: 10.5812/pedinfect.59883.

20. Moradi-Lakeh M, Esteghamati A. National Immunization Program in Iran: whys and why nots. Hum Vaccin Immunother. 2013;9(1):1124. doi: 10.4161/hv.22521. [PubMed: 23442584]. [PubMed Central: PMC3667923]. 